\title{
Possibilities for modifying risk factors for the development of hospital-acquired pneumonia in intensive care patients: results of a retrospective, observational study
}

\author{
Radovan Uvizla , Milan Kolar ${ }^{\mathrm{b}}$, Tomas Herkel ${ }^{\mathrm{a}}$, Michaela Vobrova ${ }^{\mathrm{a}}$, Katerina Langova ${ }^{\mathrm{c}}$
}

\begin{abstract}
Background. Hospital-acquired pneumonia (HAP) development is affected by a range of risk factors.
Methods. A retrospective, observational study processing data on all consecutive intensive care patients older than 18 years of age between 1 January 2011 and 31 December 2015. The aim was to determine the incidence of potential risk factors and their impact on the development of HAP.

Results. A total of 2229 patients. The overall mortality was 24.0\%; the mean APACHE II score 21.4. The mean length of ICU stay was 5.9 days and the mean length of hospital stay was 20.5 days. The criteria for HAP were met by 310 patients (13.9\%). Early- and late-onset HAP was diagnosed in 45 (14.5\%) and 265 (85.5\%) patients, respectively. The mean APACHE II score was 22.1, the mean length of ICU stay was 7.6 days and the mean length of hospital stay was 23.5 days. The most important non-modifiable factors increasing the risk of HAP were multiple organ failure (OR 13.733; $P<0.0001)$, cardiac heart disease (OR 2.255; $P<0.0001)$ and chronic renal failure (OR 2.194; $P<0.002)$. The most common modifiable factors were intolerance to enteral nutrition (OR 3.055; $P<0.0001$ ), urgent tracheal intubation (OR 1.511; $P<0.024)$, reintubation (OR 1.851; $P<0.001)$, and bronchoscopy (OR 2.558; $P<0.0001)$. Stress ulcer prophylaxis was administered to $83 \%$ of HAP patients and $68 \%$ of patients without HAP. Prophylaxis with famotidine was associated with a lower risk of HAP in $40.0 \%$ of patients (non-HAP in 49.9\%), (OR 0.669; $P=0.001$ ) than prophylaxis with pentoprazol in $42.6 \%$ and $49.5 \%$ of patients, respectively (OR $0.756 ; P=0.027$ ).

Conclusions. Factors associated with the highest risk of the development of HAP can be determined. Pharmacological prophylaxis of gastric and duodenal stress ulcers was identified as an independent risk factor for HAP.

The study was registered in the ClinicalTrials.gov database under the number NCT02779933.
\end{abstract}

Key words: hospital-acquired pneumonia, mortality, intensive care, risk factor

Received: January 13, 2017; Accepted with revision: March 31, 2017; Available online: April 26, 2017 https://doi.org/10.5507/bp.2017.019

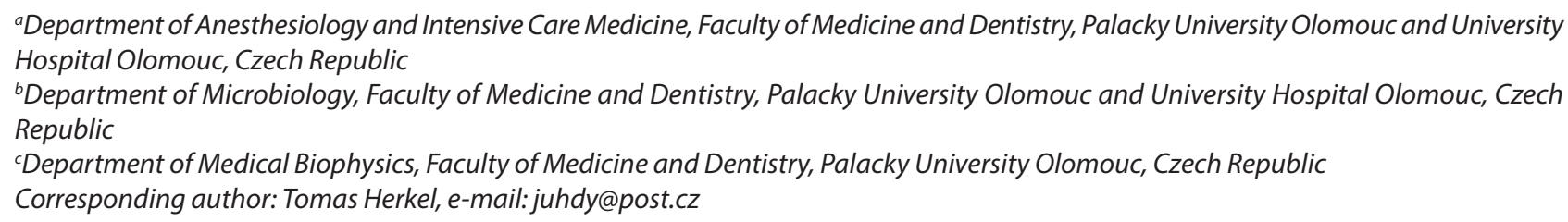

\section{INTRODUCTION}

Early and correct identification of risk factors in the nursing process with respect to the development of nosocomial bacterial infections is a prerequisite for selecting rational and safe treatments in intensive care unit (ICU) patients. Moreover, effective preventive measures, together with adequate and early empirical antibiotic therapy, contribute to successful therapy, make it shorter and less expensive ${ }^{1,2}$, and also lead to reduced mortality rates ${ }^{3}$. One of the most common infections associated with healthcare in ICU patients is hospital-acquired pneumonia (HAP). According to the American Thoracic Society and the Infectious Diseases Society of America, HAP includes ventilator-associated pneumonia (VAP) (ref. ${ }^{4}$ ). In ICU patients, HAP accounts for $10-47 \%$ of nosocomial infections ${ }^{5}$, with mortality rates ranging from $20 \%$ to $60 \%$ (ref. ${ }^{6,7}$ ). Most frequently, HAP is associated with invasive airway management and mechanical ventilation, with the latter being referred to as VAP (ref. ${ }^{8}$ ); this develops more than $48 \mathrm{~h}$ from endotracheal intubation. From an epidemiological perspective, two types of HAP are distinguished: early- and late-onset. The clinical and laboratory manifestations of early-onset HAP occur within 48 to 96 $\mathrm{h}$ from hospital admission; late-onset HAP develops from day 5 of a hospital stay but no later than 14 days after discharge ${ }^{9}$. The primary ways of transmission of etiological agents into the lower airways are most frequently microaspiration of microbes colonizing the oropharyngeal region or upper gastrointestinal tract ${ }^{7}$ or transmission of infection from the environment. The risk factors for the development of HAP may be either non-modifiable (patient-related) or modifiable (hospital-related).

The present study focused on assessing risk factors that may contribute to the development of HAP, determining their prevalence and proposing their modification in an effort to reduce the risk of developing HAP in intensive care patients. 


\section{METHODS}

\section{Setting and Study design}

A retrospective, observational study was proposed, to obtain clinical and epidemiological data on ICU patients. The sample was divided into patients who developed HAP and a subgroup of those who did not. In both subgroups, the frequency of potential risk factors for therapy and nursing care was investigated. The study was approved by the University Hospital Olomouc Ethics Committee (No. 63/16). Informed consent from patients enrolled in the study was not required. The study was registered in the ClinicalTrials.gov database under the number NCT02779933. The enrolment was influenced by neither the type of lower airway management (invasive/non-invasive) nor the result (positive/negative) of microbiological testing of samples collected from the lower airways (endobronchial aspirate or bronchoalveolar lavage) as it has been demonstrated that approximately one-third of collected samples may be microbiologically negative even if pneumonia is clinically manifested ${ }^{10}$.

\section{Participants}

Enrolled in the study were patients staying at the ICU of the Department of Anesthesiology and Intensive Care Medicine, Faculty of Medicine and Dentistry, Palacky University Olomouc and University Hospital Olomouc, between 1 January 2011 and 31 December 2015. The participants were all patients older than 18 years of age consecutively admitted to the ICU.

\section{Definitions}

Pneumonia is acute inflammation of the respiratory bronchioles, alveolar structures and pulmonary interstitium. Clinically it is defined as the presence of newly developed or progressive infiltrates on chest radiographs plus at least two other signs of respiratory tract infection: temperature $>38^{\circ} \mathrm{C}$, chest pain, purulent sputum, leukocytosis or leukopenia, signs of inflammation on auscultation, cough and/or respiratory insufficiency ${ }^{11}$. HAP is defined as pneumonia that occurs $48 \mathrm{~h}$ or more after admission, which was not incubating at the time of admission ${ }^{4}$.

\section{Outcome Assessment}

The primary outcome was investigation of the relationship between individual risk factors and the development of early- and late-onset HAP. The risk factors were assessed based on their presence in the time interval between hospital admission and the moment of fulfilling the criteria for pneumonia. The risk factors were classified into two subgroups: patient-related (non-modifiable) or hospital-related (modifiable). The patient-related factors were gender (male/female), age at enrolment (years), multiple organ failure (MOF), hypertension (HN), coronary heart disease (CHD), chronic renal failure (CRF), continuous renal replacement therapy (CRRT), acute kidney injury (AKI), diabetes mellitus (DM), chronic obstructive pulmonary disease (COPD), immunosuppression (immune) or leukopenia ( $\mathrm{WBC}<1.5 \times 10^{9} / \mathrm{L}$ ), impaired consciousness (GCS <8) and craniocerebral trauma (CCT).
The hospital-related factors were thoracotomy (thor), aspiration into the lower airways (aspir), urgent tracheal intubation (urg TI), reintubation (re TI), bronchoscopy (BSC), gastric tube (GT), intolerance of enteral nutrition (intol EN), transport outside the ICU (trans) and physiotherapy (phys).

\section{Statistical Methods}

No replacement of missing values or outliers was performed in order to minimize bias due to changed content of retrospective clinical records. Standard descriptive statistics were applied to summarize the primary data; continuous variables as means and 95\% confidence intervals or median and range; categorical variables by absolute and relative frequencies. Multivariate logistic regression was adopted for adjusting univariate results for age and for defining the final multivariate model. The selection of variables for the multivariate model was based on univariate $P<0.1$ and redundancy analysis of these preselected predictors. $P \leq 0.05$ was adopted as the level of statistical significance for all analyses. In the tables, the odds ratio (OR) with 95\% confidence interval was calculated. The statistical significance ( $P$-value) was assessed with Fisher's exact test. Factors with OR and $P$-value in bold type are statistically significant (the confidence interval does not include 1). The association of risk factors with HAP was also verified with multivariate logistic regression. As independent predictors, the model included variables with decreased $P$-value $(P<0.2)$ and risk factors present in both subgroups. The independent predictors were proton pump inhibitor (PPI), H2 antagonist (H2 antag), MOF, HN, CHD, CRF, CRRT, AKI, DM, immuno, COPD, GCS $<8$, tracheostomy (TS), CCT, thor, aspir, urg TI, re TI, BSC, GT, intol EN, trans and phys. The dependent variable was HAP (early-/late-onset). The model was constructed using the forward stepwise method involving 4 steps. SPSS 21 (IBM Corporation, 2012) was the software used.

\section{RESULTS}

\section{Patients and descriptive data}

During the above period, a total of 2229 patients, of whom 761 (34.1\%) were females and 1468 (65.9\%) were males, were admitted to an ICU for a total of 13,139 days. Their mean age was $58.7 \pm 17.2$ years (median, 63 years), specifically $62.9 \pm 18.1$ years (median, 67 years) for females and $57.5 \pm 17.4$ years (median, 62 years) for males. Their mean APACHE II score was 21.4. The mean length of ICU stay was 5.9 days and the mean length of hospital stay was 20.5 days. Based on their admission diagnosis, the participants were classified as non-surgical (1195 patients; $53.6 \%$ ) or surgical (1034 patients; $46.4 \%$ ). The overall mortality was $24.0 \%$ (535 patients irrespective of their diagnosis), of whom 170 were females and 365 were males.

The criteria for HAP were met by 310 patients (13.9\%), 108 females and 202 males. Their mean age was $60.7 \pm 17.2$ years (median, 64 years), specifically $64.9 \pm 17.8$ years (median, 68 years) for females and 59.5 
Table 1. Incidence of modifiable/non-modifiable factors for therapy and nursing care with respect to early- and late-onset HAP.

\begin{tabular}{|c|c|c|c|c|c|c|c|c|}
\hline & \multicolumn{4}{|c|}{ HAP } & \multirow{3}{*}{ OR } & & & \multirow{3}{*}{$P$} \\
\hline & \multicolumn{2}{|c|}{$\begin{array}{l}\text { early-onset } \\
(\mathrm{n}=45)\end{array}$} & \multicolumn{2}{|c|}{$\begin{array}{l}\text { late-onset } \\
(\mathrm{n}=265)\end{array}$} & & \multicolumn{2}{|c|}{$95 \% \mathrm{CI}$ for OR } & \\
\hline & $\mathrm{n}$ & $\%$ & $\mathrm{n}$ & $\%$ & & & & \\
\hline PPI & 7 & $15.6 \%$ & 125 & $47.2 \%$ & 0.206 & 0.089 & 0.479 & $<0.0001$ \\
\hline $\mathrm{H} 2$ antag & 26 & $57.8 \%$ & 98 & $37.0 \%$ & 2.332 & 1.227 & 4.431 & 0.013 \\
\hline MOF & 31 & $68.9 \%$ & 179 & $67.5 \%$ & 1.064 & 0.538 & 2.103 & 1.000 \\
\hline $\mathrm{HN}$ & 27 & $60.0 \%$ & 178 & $67.2 \%$ & 0.733 & 0.383 & 1.403 & 0.395 \\
\hline CHD & 39 & $86.7 \%$ & 135 & $50.9 \%$ & 6.259 & 2.564 & 15.282 & $<0.0001$ \\
\hline $\mathrm{CRF}$ & 9 & $20.0 \%$ & 33 & $12.5 \%$ & 1.758 & 0.777 & 3.976 & 0.236 \\
\hline CRRT & 0 & $0.0 \%$ & 66 & $24.9 \%$ & & & & $<0.0001$ \\
\hline AKI & 0 & $0.0 \%$ & 60 & $22.6 \%$ & & & & $<0.0001$ \\
\hline $\mathrm{DM}$ & 7 & $15.6 \%$ & 66 & $24.9 \%$ & 0.555 & 0.237 & 1.303 & 0.189 \\
\hline Immune & 0 & $0.0 \%$ & 27 & $10.2 \%$ & & & & 0.020 \\
\hline COPD & 18 & $40.0 \%$ & 36 & $13.6 \%$ & 4.241 & 2.122 & 8.474 & $<0.0001$ \\
\hline GCS $<8$ & 3 & $6.7 \%$ & 52 & $19.6 \%$ & 0.293 & 0.087 & 0.981 & 0.035 \\
\hline TS & 0 & $0.0 \%$ & 49 & $18.5 \%$ & & & & 0.0003 \\
\hline CCT & 0 & $0.0 \%$ & 34 & $12.8 \%$ & & & & 0.008 \\
\hline Thor & 1 & $2.2 \%$ & 30 & $11.3 \%$ & 0.178 & 0.024 & 1.340 & 0.062 \\
\hline Aspir & 0 & $0.0 \%$ & 45 & $17.0 \%$ & & & & 0.001 \\
\hline urg TI & 36 & $80.0 \%$ & 189 & $71.3 \%$ & 1.608 & 0.739 & 3.500 & 0.280 \\
\hline re TI & 13 & $28.9 \%$ & 58 & $21.9 \%$ & 1.450 & 0.715 & 2.941 & 0.338 \\
\hline BSC & 7 & $15.6 \%$ & 40 & $15.1 \%$ & 1.036 & 0.433 & 2.482 & 1.000 \\
\hline GT & 16 & $35.6 \%$ & 106 & $40.0 \%$ & 0.828 & 0.429 & 1.598 & 0.623 \\
\hline intol EN & 0 & $0.0 \%$ & 45 & $17.0 \%$ & & & & 0.001 \\
\hline Trans & 12 & $26.7 \%$ & 58 & $21.9 \%$ & 1.298 & 0.630 & 2.672 & 0.563 \\
\hline Phys & 22 & $48.9 \%$ & 44 & $16.6 \%$ & 4.804 & 2.463 & 9.371 & $<0.0001$ \\
\hline
\end{tabular}

The presence of factors in the last 7 days prior to the onset of HAP: MOF - multiple organ failure, HN - hypertension, CHD - coronary heart disease, CRF - chronic renal failure, CRRT - continuous renal replacement therapy, AKI - acute kidney injury, DM - diabetes mellitus, immuno - immunosuppression, COPD - chronic obstructive pulmonary disease, GCS $<8$ - Glasgow Coma Scale $<8$, TS - tracheostomy, CCT - craniocerebral trauma, thor - thoracotomy, aspir - aspiration into the lower airways, urg TI - urgent tracheal intubation, re TI - reintubation, BSC - bronchoscopy, GT - gastric tube, intol EN - intolerance of enteral nutrition, trans - transport outside the ICU, phys - physiotherapy

\pm 16.5 years (median, 63 years) for males. Females were statistically significantly older than males $(P=0.022)$. Early- and late-onset HAP was diagnosed in 45 (14.5\%) and $265(85.5 \%)$ patients, respectively. The flow chart is shown in Figure 1. No statistically significant relationship was found between the HAP type and patient gender $(P=1.000)$. There was a statistically significant association between the HAP type and patient age, with mean ages of 55.3 and 61.9 years for patients with earlyand late-onset HAP, respectively $(P=0.049)$. The mean APACHE II score was 22.136 (range, 8-43; median, 21.0), specifically 23.706 (range, 8-43; median, 24.0) for females and 21.704 (range, 8-40; median, 21.0) for males. The difference between gender was not statistically sig-

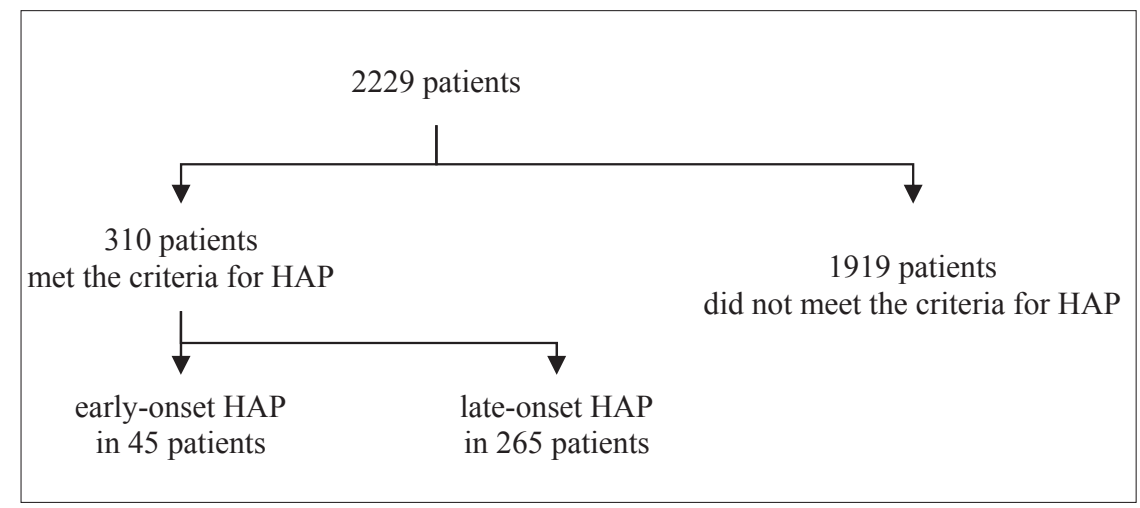

Fig. 1. Study flow chart. 
Table 2. Incidence of modifiable/non-modifiable factors for therapy and nursing care with respect to the presence/absence of HAP.

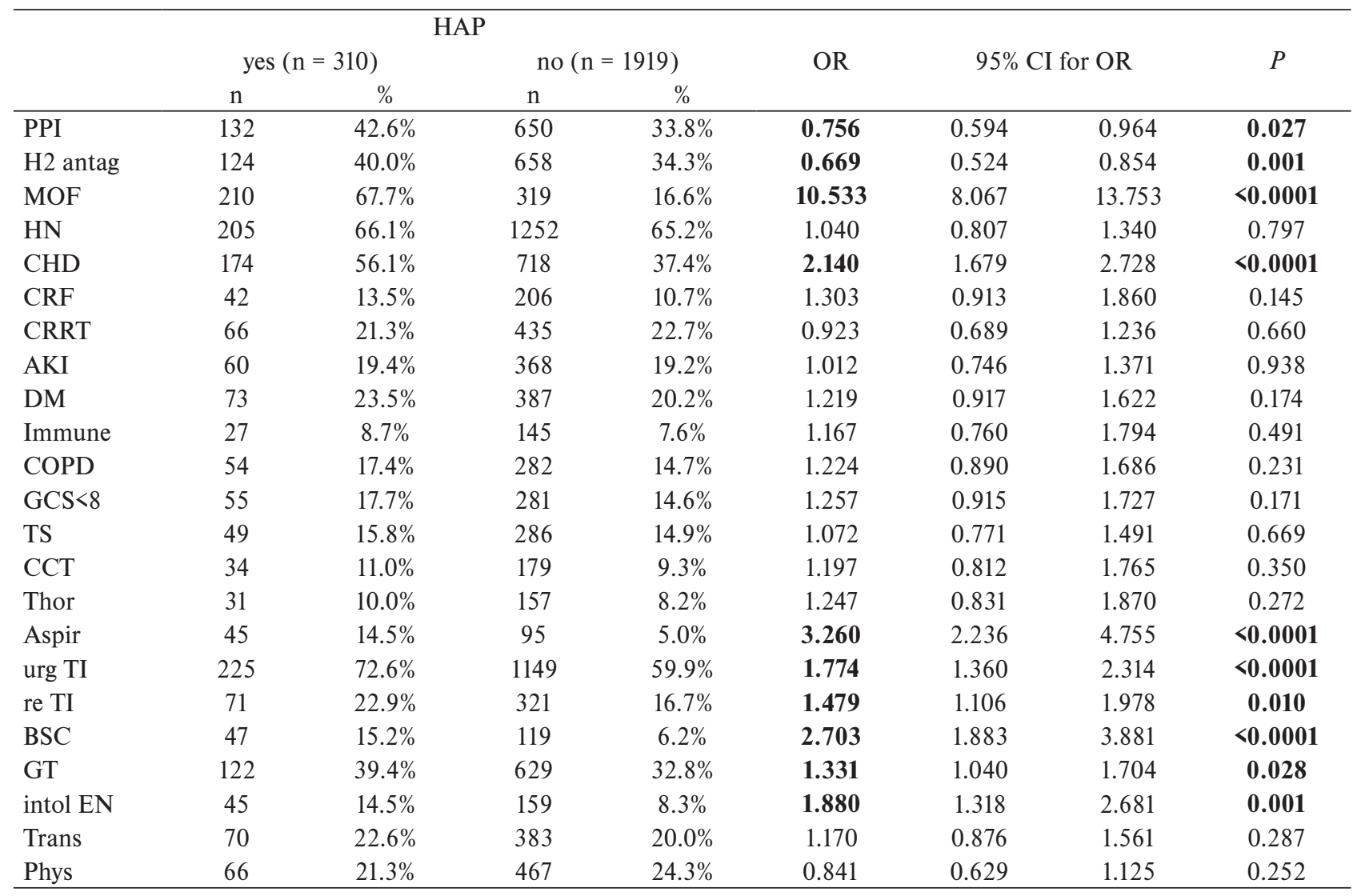

The presence of factors in the last 7 days prior to the onset of HAP: PPI - administration of the proton pump inhibitor pantoprazole in therapeutic doses, $\mathrm{H} 2$ antag - the $\mathrm{H} 2$ antagonist famotidine at therapeutic doses, MOF - multiple organ failure, HN - hypertension, CHD - coronary heart disease, CRF - chronic renal failure, CRRT - continuous renal replacement therapy, AKI - acute kidney injury, DM - diabetes mellitus, immuno - immunosuppression, COPD - chronic obstructive pulmonary disease, GCS < 8 - Glasgow Coma Scale < 8, TS - tracheostomy, CCT - craniocerebral trauma, thor - thoracotomy, aspir - aspiration into the lower airways, urg TI - urgent tracheal intubation, re TI - reintubation, BSC - bronchoscopy, GT - gastric tube, intol EN - intolerance of enteral nutrition, trans - transport outside the ICU, phys - physiotherapy

nificant $(P=0.062)$. No association was found between the APACHE II score and HAP type. The mean length of HAP patients' stay in the ICU was 7.6 days and their mean hospital stay was 23.5 days.

\section{Main results}

The absolute and relative frequencies of modifiable/ non-modifiable factors for therapy and nursing care with respect to the risk of developing early- and late-onset HAP are shown in Table 1.

Stress ulcer prophylaxis was administered to $83 \%$ of HAP patients and $68 \%$ of patients without HAP.

The absolute and relative frequencies of modifiable/ non-modifiable factors for therapy and nursing care with respect to the presence/absence of HAP are shown in Table 2.

After the relationships between therapy / nursing care factors and the risk of the development of HAP were assessed by multivariate logistic regression, statistically significant predictors for the development of HAP were identified as shown in Table 3.
Table 3. Statistically significant independent predictors for the development of HAP.

\begin{tabular}{lrrrc}
\hline & OR & \multicolumn{2}{c}{$95 \%$ CI for OR } & $P$ \\
\hline PPI & 0.010 & 0.005 & 0.022 & $<0.0001$ \\
H2 antag & 0.012 & 0.006 & 0.027 & $<0.0001$ \\
MOF & 13.733 & 9.966 & 18.923 & $<0.0001$ \\
CHD & 2.255 & 1.645 & 3.092 & $<0.0001$ \\
CRF & 2.194 & 1.344 & 3.580 & 0.002 \\
urg TI & 1.511 & 1.056 & 2.160 & 0.024 \\
re TI & 1.851 & 1.273 & 2.690 & 0.001 \\
BSC & 2.558 & 1.610 & 4.065 & 0.0001 \\
intol EN & 3.055 & 1.962 & 4.757 & $<0.0001$ \\
\hline
\end{tabular}

The presence of factors in the last 7 days prior to the onset of HAP: MOF - multiple organ failure, CHD - coronary heart disease, CRF chronic renal failure, urg TI - urgent tracheal intubation, re TI - reintubation, BSC - bronchoscopy, intol EN - intolerance of enteral nutrition 


\section{DISCUSSION}

Data are presented from a long-term study of a large sample of ICU patients comparing the risk posed by individual factors of therapy and nursing care with respect to the development of early- and late-onset HAP; the impact that the two most common types of stress ulcer prophylaxis have on the incidence of HAP is also documented. The study showed that certain factors, both modifiable and non-modifiable, significantly increase the risk of HAP. The most important non-modifiable factors increasing the risk of developing HAP are MOF, CHD and, at a lower level of statistical significance, the presence of CRF. Patient-related risk factors were investigated, among others, in a large study of 8657 ICU patients which identified atrial fibrillation as a significant risk factor for the development of HAP. However, gender, smoking, CHD, DM, rheumatic heart disease, non-rheumatic valvular disease, myocardiopathy/myocarditis, hyperlipidemia, electrolyte disturbance and congenital heart disease were not significant risk factors for HAP (ref. $\left.{ }^{12}\right)$. Another study of ICU patients with HAP in association with Staphylococcus aureus showed that significant risk factors are diseases such as liver cirrhosis or DM. On the other hand, the study failed to show a relationship to COPD, hypertension or CRF (ref. ${ }^{13}$ ). Consistent with the present study, Vardakas et al. did not identify DM as a risk factor for HAP $\left(\right.$ ref. $\left.{ }^{14}\right)$. Finally, monitoring of residual gastric volume was not a factor significantly reducing the risk of developing HAP $\left(\right.$ ref. $\left.{ }^{15}\right)$.

Among hospital-related, or modifiable, factors included in the present study, intolerance of enteral nutrition was the most significant, with urgent tracheal intubation, reintubation and bronchoscopy showing a lower level of statistical significance. If well-tolerated, enteral nutrition is not a risk factor. This was documented, for example, in a study of polytrauma patients showing that enteral nutrition can decrease the incidence of nosocomial pneumonia ${ }^{16}$. By contrast, the presence of an inserted GT is considered as a significant risk factor, as seen from a recent large study of 4427 patients documenting that mechanical ventilation and the use of a GT were the most significant risk factors for the development of HAP $\left(\right.$ ref. $\left.{ }^{17}\right)$. Apart from the insertion of a GT, patient immobility is a stronger risk factor for HAP than dysphagia, as shown by Brogan et al. ${ }^{18}$. Also consistent with the present study are the results of a Polish study of 1227 ICU patients showing a statistically significant correlation between the development of HAP and incidence of reintubation, tracheostomy and bronchoscopy 19. Another study showed the effect of a history of pre-hospital aspiration or the presence of blood and emesis in the airways after intubation on the development of HAP (aspiration $16 \%$ vs. no aspiration $4 \%)\left(\right.$ ref. $\left.^{20}\right)$. In the HAP group $10 \%$ of patients and in the non-HAP group $8 \%$ of patients had undergone thoracic surgery; the difference was not statistically significant and therefore the present study failed to identify thoracic surgery as a risk factor for HAP. Some studies referred to the incidence of pneumonia and subsequently of thoracic surgery in $3.3-25 \%$. Similarly, a study of 604 patients undergoing resection of bronchogenic carcinoma showed 5\% incidence of HAP (ref. ${ }^{21}$ ). In a group of major heart surgery patients, however, the incidence of HAP was $46 \%$ in those requiring more than $48 \mathrm{~h}$ of mechanical ventilation. The independent risk factors for HAP were age older than 70 years, perioperative transfusions, days of mechanical ventilation, reintubation, previous cardiac surgery, emergent surgery and intraoperative inotropic support ${ }^{22}$. In the present study, previous aspiration was only a risk factor at a low level of statistical significance. Craniocerebral trauma or neurosurgical intervention were associated with the development of HAP in $13 \%$ of patients in the present study. In neurosurgery patients, univariate analysis demonstrated that a low GCS, long hospital stay, use of wide-spectrum antibiotics, mechanical ventilation, total parenteral nutrition and reoperation were risk factors for nosocomial infections ${ }^{23}$. Similarly, in abdominal surgery patients, an ICU stay longer than or equal to 7 days and a postoperative hospital stay of 15 days or more were the predictive factors most strongly associated with lung infection $^{24}$. The varied results for individual risk factors may also be documented by one study on the incidence of HAP in non-ICU patients. Malnutrition, CRF, anemia, depression of consciousness, previous hospitalization and thoracic surgery were significant risk factors for HAP in these patients ${ }^{25}$. There were lower mean ages of 55.3 and 61.9 years for patients with early- and lateonset HAP, respectively. We explain that the late-onset HAP affects more weakened patients with polymorbidity, who are more susceptible to infections caused by MDR pathogens. Further, in the presented study bronchoscopy was associated with a higher incidence of HAP, but bronchoscopy cannot be considered as a risk factor for HAP, because risk factors are the reasons which led to its implementation. These were most commonly: massive congestion, aspiration into the lungs, chronic lung disease or esophagotracheal fistula. Also intolerance of enteral nutrition is associated with higher incidence of HAP more due to capillar action from around the gastric tube and more frequent gastric fluid retention in the space above the obturation balloon of the tracheal tube causes silent microaspiration.

An important outcome of the present study is assessment of the impact of the two most common types of stress ulcer prophylaxis on the incidence of HAP. Stress ulcer prophylaxis was administered to $83 \%$ of HAP patients and $68 \%$ of patients without HAP. Prophylaxis with famotidine used to prevent stress ulcer was associated with a lower risk of HAP in $40 \%$ of patients (OR $0.669 ; P=0.001$ ) than pentoprazol prophylaxis in $43 \%$ of patients (OR 0.756; $P=0.027$ ), but the results are not as significant as in another similar study. The rate of HAP was lower for an $\mathrm{H} 2$ antag (10\%) than for a PPI (30\%). Administration of the $\mathrm{H} 2$ antag was also associated with fewer hospital days (5.6 vs. 17.6) (ref. ${ }^{26}$ ). A statistically significant difference in the incidence of HAP was found in a study comparing the effects of sucralfate (14\%) and 
a PPI (36\%) (ref. $\left.{ }^{27}\right)$. Similar findings were also reported by authors of a large retrospective study of 21.214 cardiac surgery ICU patients, with the incidence of HAP being higher in patients receiving a PPI as compared to an $\mathrm{H} 2 \operatorname{antag}^{28}$. However, the administration of stress ulcer prophylaxis itself is linked to a higher risk of HAP, as documented, for example, by a large study of 63.878 patients showing that acid-suppressive medication was associated with a higher incidence of HAP, the association being significant for a PPI and non-significant for H2 $\operatorname{antag}^{29}$.

The present study found differences in the incidence of certain modifiable/non-modifiable factors for early- and late-onset HAP (Table 1). In early-onset HAP, the statistically significantly more frequent factors were CHD, COPD and phys, while in late-onset HAP, AKI, CRRT and TS were statistically significantly more common. However, the presence of physiotherapy in early-onset HAP is not considered a factor increasing its incidence. This is rather associated with physiotherapy provided to at-risk patients who subsequently develop HAP. Similarly, higher TS rates in patients with late-onset HAP is considered a sign of more severe pneumonia requiring longer ventilator use. TS was performed in patients prior to randomization, prior to formation of HAP and the reason for its implementation was different than the current HAP attack. Most often it was longterm impairment of consciousness, respiratory insufficiency after previous severe pneumonia or long term ventilator dependence in chronic pulmonary disease patients. The presence of TS increases the HAP incidence probably due to the bypassed upper airway, as a natural bacterial filter.

\section{CONCLUSION}

Epidemiological data on ICU patients obtained over the five-year period show that the highest risk of HAP is associated with the patient-related factors MOF, CHD and CRF and the following hospital-related factors: urgent tracheal intubation, reintubation, bronchoscopy and intolerance of enteral nutrition. Additionally, stress ulcer prophylaxis was found to be an independent risk factor for the development of HAP. Prophylaxis with famotidine was associated with a lower risk than pentoprazol prophylaxis.

\section{ABBREVIATIONS}

AKI, acute kidney injury; APACHE II, Acute Physiology and Chronic Health Evaluation II; BSC, bronchoscopy; ICU, intensive care unit; CHD, coronary heart disease; COPD, chronic obstructive pulmonary disease; CRRT, continuous renal replacement therapy; DM, diabetes mellitus; EN, enteral nutrition; GCS, Glasgow Coma Scale; GT, gastric tube; HAP, hospital-acquired pneumonia; HN, hypertension; CRF, chronic renal failure; CRRT, continuous renal replacement therapy; ICU, intensive care unit; MOF, multiple organ failure; PPI, proton pump inhibitor; TI, tracheal intubation; TS, tracheostomy; VAP, ventilatorassociated pneumonia; WBC, white blood cell count.
Author contributions: RU, TH, MK: manuscript writing; RU, TH: study design; RU, MK, TH, MV: analysis and interpretation of data; RU, TH, MV: drafting the manuscript and revising it critically for important intellectual content; RU, TH, MK: agreed to be accountable for all aspects of the work in ensuring that queries relating to the accuracy and integrity of the work are appropriately investigated and resolved; RU, TH, MK, MV: final approval.

Conflict of interest statement: None declared.

\section{REFERENCES}

1. Joseph NM, Sistla S, Dutta TK, Badhe AS, Parija SC. Ventilatorassociated pneumonia: a review. Eur J Intern Med 2010;21:360-8.

2. Jones, RN. Microbial etiologies of hospital-acquired pneumonia and ventilator-associated bacterial pneumonia. Clin Index Dis 2010;51(1 Suppl):81-7.

3. Rello J, Torres A, Ricart M, Valles J, Gonzales J, Artigas A, RodriquezRoisin R. Ventilator-associated pneumonia by Staphylococcus aureus. Comparison of methicillin-resistant and methicillin-sensitive episodes. Am J Respir Crit Care Med 1994;150:1545-9.

4. American Thoracic Society, Infectious Diseases Society of America. Guidelines for the management of adults with hospital-acquired, ventilator-associated, and healthcare-associated pneumonia. Am J Respir Crit Care Med 2005;171:388.

5. Vincent JL, Bihari DJ, Suter PM, Bruining HA, White J, NicolasChanoin MH, Wolff M, Spencer RC, Hemmer M. The prevalence of nosocomial infection in intensive care units in Europe. Results of the European Prevalence of Infection in Intensive Care (EPIC) Study. EPIC International Advisory Committee. JAMA 1995;274(8):639-44.

6. Guideline for prevention of nosocomial pneumonia. Centers for Disease Control and Prevention. Respir Care 1994;39:1191-236.

7. File TM Jr, Bartlett JG, Thorner AR. Epidemiology, pathogenesis, microbiology, and diagnosis of hospital-acquired, ventilator-associated pneumonia in adults. www.uptodate.com

8. Ibrahim EH, Ward S, Sherman G, Kollef MH. A comparative analysis of patients with early-onset vs late-onset nosocomial pneumonia in the ICU setting. Chest 2000;117(5):1434-42.

9. Langer M, Cigada M, Mandelli M, Mosconi P, Tognoni G. Early onset pneumonia: a multicenter study in intensive care units. Intensive Care Med 1987;13:342-6.

10. GabrhelikT, Hanulik V, Jakubec P, Uvizl R, Doubravska L, Roderova M, Adamus M, Kolek V, Kolar M. Validity comparison of various biological samples from lower airway and their contribution for the detection of nosocomial pneumonia etiological agents. Klin Mikrobiol Infekc Lek 2015;21(1):4-9.

11. Kolek V, Kasak V, Vasakova M. Pneumologie. 2nd edition. Maxdorf;.2014:195-210.

12. Zhu J, Zhang X, Shi G, Yi K, Tan X. Atrial Fibrillation Is an Independent Risk Factor for Hospital-Acquired Pneumonia. PLoS One. 2015;10(7):e0131782.

13. Wu HP, Chu CM, Lin CY, Yu CC, Hua CC, Yu TJ, Liu YC. Liver Cirrhosis and Diabetes Mellitus Are Risk Factors for Staphylococcus aureus Infection in Patients with Healthcare-Associated or HospitalAcquired Pneumonia. Pulm Med 2016;2016:4706150. doi: $10.1155 / 2016 / 4706150$

14. Vardakas KZ, Siempos II, Falagas ME. Diabetes mellitus as a risk factor for nosocomial pneumonia and associated mortality. Diabet Med 2007;24(10):1168-71.

15. Reignier J, Mercier E, Le Gouge A, Boulain T, Desachy A, Bellec F, Clavel M, Frat JP, Plantefeve G, Quenot JP, Lascarrou JB. Effect of not monitoring residual gastric volume on risk of ventilator-associated pneumonia in adults receiving mechanical ventilation and early enteral feeding: a randomized controlled trial. Clinical Research in Intensive Care and Sepsis (CRICS) Group. JAMA 2013;309(3):249-56.

16. Kompan L, Vidmar G, Spindler-Vesel A, Pecar J. Is early enteral nutrition a risk factor for gastric intolerance and pneumonia? Clin Nutr 2004;23(4):527-32.

17. Guzmán-Herrador B, Molina CD, Allam MF, Navajas RF. Independent risk factors associated with hospital-acquired pneumonia in an adult 
ICU: 4-year prospective cohort study in a university reference hospital. J Public Health (Oxf) 2016;38(2):378-83.

18. Brogan E, Langdon C, Brookes K, Budgeon C, Blacker D. Respiratory infections in acute stroke: nasogastric tubes and immobility are stronger predictors than dysphagia. Dysphagia 2014;29(3):340-5

19. Wałaszek M, Kosiarska A, Gniadek A, Kołpa M, Wolak Z, Dobroś W, Siadek J. The risk factors for hospital-acquired pneumonia in the Intensive Care Unit. Przegl Epidemiol 2016;70(1):107-10.

20. Fawcett VJ, Warner KJ, Cuschieri J, Copass M, Grabinsky A, Kwok H Rea T, Evans HL. Pre-hospital aspiration is associated with increased pulmonary complications.. Surg Infect (Larchmt) 2015;16(2):159-64.

21. Diaz-Ravetllat V, Ferrer M, Gimferrer-Garolera JM, Molins L, Torres A. Risk factors of postoperative nosocomial pneumonia after resection of bronchogenic carcinoma. Respiratory Medicine 2012;106:146371.

22. Hortal J, Giannella M, Barrio JM, Desco M, Bouza E, Muñoz P. Incidence and risk factors for ventilator-associated pneumonia after major heart surgery. Intensive Care Med 2009;35:1518-25.

23. Gomez C, Celik F, Tekin R, Kamasak K, Turan Y, Palancı Y, Bozkurt F, Bozkurt M. Evaluation of risk factors affecting hospital-acquired infections in the neurosurgery Intensive Care Unit. Int J Neurosci 2014;124(7):503-8.
24. Evaristo-Méndez G, Rocha-Calderón C.H. Risk factors for nosocomial pneumonia in patients with abdominal surgery. Cirugía y Cirujanos 2016;84(1):21-7.

25. Sopena N, Heras E, Casas I, Bechini J, Guasch I, Pedro-Botet ML, Roure $S$, Sabrià M. Risk factors for hospital-acquired pneumonia outside the intensive care unit: A case-control study. American Journal of Infection Control 2014;42:38-42.

26. Bashar FR, Manuchehrian N, Mahmoudabadi M, Hajiesmaeili MR, Torabian S. Effects of ranitidine and pantoprazole on ventilatorassociated pneumonia: a randomized double-blind clinical trial. Tanaffos 2013;12(2):16-21.

27. Khorvash F, Abbasi S, Meidani M, Dehdashti F, Ataei B. The comparison between proton pump inhibitors and sucralfate in incidence of ventilator associated pneumonia in critically ill patients. Adv Biomed Res 2014;3:52. doi: 10.4103/2277-9175.125789

28. Bateman BT, Bykov K, Choudhry NK, Schneeweis S, Gagne JJ, Polinski JM, Franklin JM, Doherty M, Fischer MA, Rassen JA. Type of stress ulcer prophylaxis and risk of nosocomial pneumonia in cardiac surgical patients. BMJ 2013;347:5416. doi: 10.1136/bmj.f5416

29. Herzig SJ, Howell MD, Ngo LH, Marcantonio ER. Acid-Suppressive Medication Use and the Risk for Hospital-Acquired Pneumonia. JAMA 2009;301(20):2120-8. 